\title{
Explicit representation of extended Jones matrix for oblique light propagation through a crystalline slab
}

\author{
${ }^{1}$ Nastishin Yu. A. and ${ }^{2}$ Nastyshyn S. Yu. \\ ${ }^{1}$ Institute of Physical Optics, 23 Dragomanov St., 79005 Lviv, Ukraine \\ e-mail: Nastishin@ifo.lviv.ua \\ 2 Physics Department, Ivan Franko National University of Lviv, \\ 6 Kyrylo and Methodiy St., 79005 Lviv, Ukraine
}

Received: 03.11.2011

\begin{abstract}
We derive compact representation of an extended Jones matrix (EJM) suggested by Lien [Lien A, 1997. Liq. Cryst. 22: 171-175] for the case of oblique light incidence. Our representation of the EJM is handy for analytical calculations and has the form of a general Jones matrix, with nonzero imaginary parts of its offdiagonal elements. An analogy between these two matrices is discussed. For a crystal slab with the optic axis along the slab normal, our representation of the EJM reduces to a form that yields the Jones vector identical to that obtained by the other authors in the frame of paraxial approximation.
\end{abstract}

Keywords: Jones matrix formalism, extended Jones matrix, oblique light incidence, linear birefringence, Jones birefringence

PACS: 42.25.Bs, 42.25.Ja, 42.25.Lc, 78.20.Bh

UDC: $535.3,535.5$

\section{Introduction}

Jones matrix formalism is a powerful tool for calculating electric field vector of a light wave exiting a system of optical elements. Originally, it has been designed for normal light incidence [1-7]. Several attempts have been made to extend this method for the case of oblique incidence. At first Yeh $[8,9]$, and then Lien $[10,11]$ have derived an extended Jones matrix (EJM) for the oblique incidence of light. Though these two approaches differ in the way of matching the boundary conditions and so yield literally different forms of the EJM, they produce reasonable agreement when applied for numerical calculations of the light transmittance through twisted nematic liquid-crystalline cells. Lien has neglected the effect of light reflection and considered boundary conditions only for the electric field vector $[10,11]$. As a result, his version of the EJM looks simpler when compared with that by Yeh $[8,9]$ and because of this is extensively used for simulating liquid-crystal displays. Yu and Kwok [12] have compared the two approaches to the results obtained using the fast $4 \times 4$ Berreman's matrix method [13] (which is an approximation of the exact Berreman's $4 \times 4$ matrix method $[14,15])$ and have shown that the Yeh's version $[8,9]$ produces smaller errors. Later on, Lien [16] has suggested a new version of the EJM obtained when combining the boundary conditions for the both electric and magnetic field vectors. This new algorithm indeed shows some improvement [12] but becomes twice as lengthy. Whether or not, but at the incidence angles smaller than $45^{\circ}$, the Lien's version $[10,11]$ still shows good enough accuracy, in comparison to the Yeh's approach.

Since we seek for a compact representation of the EJM convenient for analytical derivations, in this work we will deal with the older (less exact but essentially simpler) version of the EJM derived by Lien $[10,11]$. It takes the form

$$
J_{L}=S G_{L} S^{-1}
$$


where

$$
G_{L}=\left[\begin{array}{cc}
e^{i k_{11} z} & 0 \\
0 & e^{i k_{22} z}
\end{array}\right] .
$$

Here $J_{L}$ is written in the right-hand Cartesian coordinate system, of which $Z$ axis lies along the normal to the surfaces of crystalline slab and $X$ axis is directed along the projection of the light wave vector $\vec{k}$ on the surface first met by the light beam (this is called below as a $k$-coordinate system).

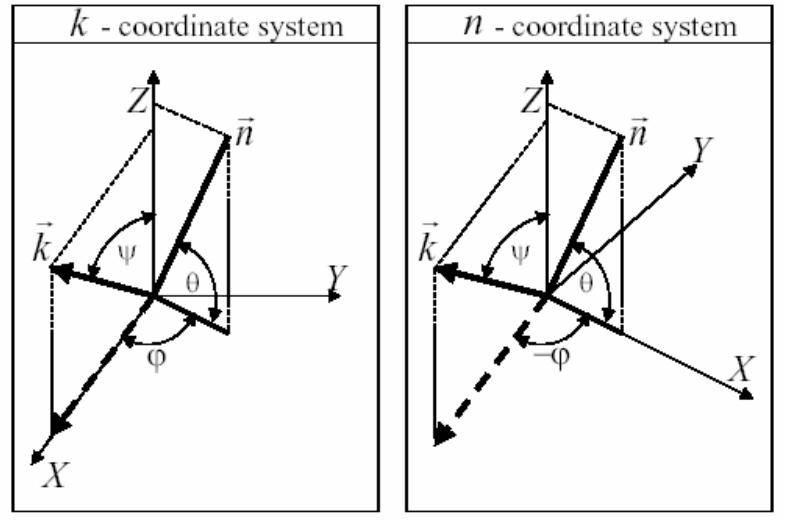

Fig. 1. A sketch of $k$ - and $n$ - coordinate systems.

Of the other notation, the subscript $L$ refers to the conventions of matrices adopted by Lien in Ref. [11], and the matrix $S$ plays a part of rotation matrix. The latter transforms the birefringence Jones matrix $G_{L}$ (designed for the normal light incidence and written in the principal coordinate system) into the matrix $J_{L}$ playing a part of the Jones matrix relating the incident and exit Jones vectors at the oblique light propagation. Let us remind that $J_{L}$ is written in the $k$-coordinate system. However, $S$ does not represent a true rotation matrix because it is not orthogonal, being also called as "non-orthonormal".

The property of non-orthogonality for the $S$ matrix reflects the fact that, in a general case, the ordinary and extraordinary waves at the oblique light incidence propagate in crystal along different directions defined by the Snell's law of refraction at the crystal interface and thus are not mutually orthogonal [8]. For further details we refer a reader to Ref. [11], where a detailed derivation of Eq. (1) is given. The components of the matrix $S$ written in the $k$-coordinate system with the light wave vector $\vec{k}=k_{0}(\sin \psi, 0$; $\cos \psi)$ fixed in the $Z X$ plane (with $\psi$ being the light incidence angle, $k_{0}=2 \pi / \lambda$, and $\lambda$ the light wavelength in vacuum) are of the following form [11]:

$$
\begin{aligned}
& S_{11}=\left[\left(\frac{k_{z 1}}{k_{0}}\right)^{2}+\left(\frac{k_{x}}{k_{0}}\right)^{2}-\varepsilon_{y y}\right]\left[\left(\frac{k_{x}}{k_{0}}\right)^{2}-\varepsilon_{z z}\right]-\varepsilon_{y z} \varepsilon_{z y}, \\
& S_{12}=\left[-\left(\frac{k_{x}}{k_{0}}\right)^{2}+\varepsilon_{z z}\right] \varepsilon_{x y}-\left(\frac{k_{x}}{k_{0}} \frac{k_{z 2}}{k_{0}}+\varepsilon_{x z}\right) \varepsilon_{z y}, \\
& S_{21}=\left[\left(\frac{k_{x}}{k_{0}}\right)^{2}-\varepsilon_{z z}\right] \varepsilon_{y x}+\left(\frac{k_{x}}{k_{0}} \frac{k_{z 1}}{k_{0}}+\varepsilon_{z x}\right) \varepsilon_{y z}, \\
& S_{22}=\left[-\left(\frac{k_{z 2}}{k_{0}}\right)^{2}+\varepsilon_{x x}\right]\left[\left(\frac{k_{x}}{k_{0}}\right)^{2}-\varepsilon_{z z}\right]+\left(\frac{k_{x}}{k_{0}} \frac{k_{z 2}}{k_{0}}+\varepsilon_{z x}\right)\left(\frac{k_{x}}{k_{0}} \frac{k_{z 2}}{k_{0}}+\varepsilon_{x z}\right),
\end{aligned}
$$


where

$$
k_{z 1}=k_{0} \sqrt{n_{o}^{2}-\left(\frac{k_{x}}{k_{0}}\right)^{2}}
$$

and

$$
k_{z 2}=k_{0}\left[-\frac{\varepsilon_{x z}}{\varepsilon_{z z}} \frac{k_{x}}{k_{0}}+\frac{n_{o} n_{e}}{\varepsilon_{z z}} \sqrt{\varepsilon_{z z}-\left(1-\frac{n_{e}^{2}-n_{o}^{2}}{n_{e}^{2}} \cos ^{2} \theta \sin ^{2} \varphi\right)\left(\frac{k_{x}}{k_{0}}\right)^{2}}\right]
$$

are $z$-components of the light wave vectors for the two eigenwaves propagating in crystal slab, and $\varepsilon_{i j}$ denote the components of the dielectric tensor of that slab:

$$
\left\{\begin{array}{l}
\varepsilon_{x x}=n_{o}^{2}+\left(n_{e}^{2}-n_{o}^{2}\right) \cos ^{2} \theta \cos ^{2} \varphi \\
\varepsilon_{x y}=\varepsilon_{y x}=\left(n_{e}^{2}-n_{o}^{2}\right) \cos ^{2} \theta \sin \varphi \cos \varphi \\
\varepsilon_{x z}=\varepsilon_{z x}=\left(n_{e}^{2}-n_{o}^{2}\right) \sin \theta \cos \theta \cos \varphi \\
\varepsilon_{y y}=n_{o}^{2}+\left(n_{e}^{2}-n_{o}^{2}\right) \cos ^{2} \theta \sin ^{2} \varphi \\
\varepsilon_{y z}=\varepsilon_{z y}=\left(n_{e}^{2}-n_{o}^{2}\right) \sin \theta \cos \theta \sin \varphi \\
\varepsilon_{z z}=n_{o}^{2}+\left(n_{e}^{2}-n_{o}^{2}\right) \sin ^{2} \theta
\end{array} .\right.
$$

Here $\theta$ and $\varphi$ stand for the polar and azimuthal angles of the optic axis, $n_{o}$ and $n_{e}$ the refractive indices for the ordinary and extraordinary light waves. The angles $\theta$ and $\varphi$ are measured with respect to the $X Y$ plane and the $X$ axis, respectively.

The equation system given by Eqs. (1)-(6) is applicable for uniform optically uniaxial nematic cells or single-crystalline plates. For a distorted nematic cell, the liquid-crystal slab is sliced into thin plates such that each plate is characterised by its own orientation of the optic axis being uniform within the plate. The Jones matrix of deformed nematic would then be a matrix product of the matrices of the elementary plates. Such an approach works well when the state of exiting light is calculated numerically with a computer. However, the analytical calculations are difficult because of lengthy representation of the matrix components (see Eqs. (1)-(6)). For the Yeh's $[8,9]$ and the new Lien's [16] approaches they are even more routine.

The article is organised as follows. In Section 2 we present a compact form of the matrix $S$ handy for analytical calculations. Further substituting our representation for $S$ into Eq. (1), we find that the obtained EJM has a form of a general Jones matrix (GJM) $M$ [7] having nonzero imaginary parts of the off-diagonal elements $\left(M_{12} \neq M_{21}\right)$. This is what can be expected intuitively when the light reflection is neglected. Considering that the GJM is represented in the principal coordinate system (a so-called $n$-coordinate system, in which the $Z$ axis is directed along the normal to crystal slab surfaces and the $X$ axis along the projection of the optic axis $\vec{n}$ on the first surface) and the EJM is written in the $k$-coordinate system, in Section 3 we transform the EJM from the $k$-coordinate system to the principal $n$-coordinate system, in order to compare these two matrices. We find that even after this transformation the EJM has non-zero imaginary parts in its off-diagonal components. In Section 4 we discuss an analogy between the EJM and the GJM. Section 5 demonstrates that for a homeotropic liquid-crystalline cell, with its optic axis $\vec{n}$ perpendicular to the substrates, the EJM reduces to a form obtained earlier by the other authors (see [17] and references herein) in a paraxial approximation. For a circularly polarised beam of 
conical rays and under the conditions of specific experimental geometry, this leads to the electric field components degenerated with respect to the azimuthal angle of the incident ray plane, thus being at the heart of optical singularities (or wave dislocations, a term coined by Nye and Berry [18] - see also [19]). Finally, Section 6 concludes our work.

\section{Compact representation of matrix $S$}

After substituting Eqs. (6) into Eqs. (3) and some algebra, we find that $S$ reduces to a compact form (cf. with initial Eqs. (3)-(6)) $S=R_{L}(\theta, \varphi)\left(n_{e}^{2}-n_{o}^{2}\right) \cos \theta \sin \varphi$, with

$$
R_{L}(\theta, \varphi)=\left[\begin{array}{cc}
k_{1}^{2} \cos \theta \sin \varphi & k_{1}^{2} \cos \theta \cos \varphi-k_{x 0} k_{2} \sin \theta \\
-k_{1}^{2} \cos \theta \cos \varphi+k_{x 0} k_{1} \sin \theta & n_{o}^{2} \cos \theta \sin \varphi
\end{array}\right]
$$

and $k_{1}=k_{z 1} / k_{0}, k_{2}=k_{z 2} / k_{0}, k_{x 0}=k_{x} / k_{0}=\sin \psi$. A common factor $\left(n_{e}^{2}-n_{o}^{2}\right) \cos \theta \sin \varphi$ in $S$ can be omitted because it cancels when being substituted into Eq. (1). For the normal incidence $\left(k_{x 0}=0\right), R_{L}(\theta, \varphi)$ reduces to $\left.R_{L}\right|_{k_{k=0}=0}=n_{0}^{2} \cos \theta R_{0 L}(\varphi)$, where

$$
R_{0 L}(\varphi)=\left[\begin{array}{cc}
\sin \varphi & \cos \varphi \\
-\cos \varphi & \sin \varphi
\end{array}\right] .
$$

The form of $R_{0 L}(\varphi)$ differs from the standard representation of the rotation matrix [20],

$$
R_{0}(\varphi)=\left[\begin{array}{cc}
\cos \varphi & -\sin \varphi \\
\sin \varphi & \cos \varphi
\end{array}\right]
$$

The origin of this difference is in the form of the birefringent matrix $G_{L}$ (see Eq. (2)) used in [11]. As stated in the Section 1, one of the goals of this article is to compare the form of the EJM to the general form of the Jones matrix given by Eqs. (3.26) and (4.17) of Ref. [7]. To do this one has to match the notation conventions used in [11] to those by Jones in Ref. [7]. The standard form of the birefringent matrix introduced by Jones would read as

$$
G_{0}=\left[\begin{array}{cc}
e^{-i k_{z 2} z} & 0 \\
0 & e^{-i k_{z 1} z}
\end{array}\right],
$$

in accordance with the expression

$$
E=E_{0} e^{i(\omega t-k z)}
$$

for the electric field vector for light propagating in an isotropic medium. It is assumed that the medium has the absorption index $\kappa$ and the refractive index $n$, the $X Y Z$ coordinate system is right-handed, and the light with the amplitude $E_{0}$ and the wave number $k=2 \pi(\kappa+i n) / \lambda$ travels in the positive direction of the $Z$ axis. Below we adopt the convention corresponding to the form of the electric field vector given by Eq. (11), thus replacing $z$ by $-z$ in Eq. (2). The matrix $G_{L}$ in which $z$ is replaced by $-z$ will be denoted $G$. The $G$ matrix can be obtained from $G_{0}$ after rotating it by $\pi / 2$ :

$$
G=R_{0}\left(\frac{\pi}{2}\right) G_{0} R_{0}\left(-\frac{\pi}{2}\right) .
$$

Then the EJM adopted to the Jones notation convention reads as

$$
J=R G_{0} R^{-1},
$$

where 


$$
R=S R_{0}\left(\frac{\pi}{2}\right)=\left[\begin{array}{ll}
S_{11} & S_{12} \\
S_{21} & S_{22}
\end{array}\right]\left[\begin{array}{cc}
0 & -1 \\
1 & 0
\end{array}\right]=\left[\begin{array}{ll}
S_{12} & -S_{11} \\
S_{22} & -S_{21}
\end{array}\right] .
$$

Explicitly $R$ acquires the following form:

$$
R=\left[\begin{array}{cc}
k_{1}^{2} \cos \theta \cos \varphi-k_{x 0} k_{2} \sin \theta & -k_{1}^{2} \cos \theta \sin \varphi \\
n_{o}^{2} \cos \theta \sin \varphi & k_{1}^{2} \cos \theta \cos \varphi-k_{x 0} k_{1} \sin \theta
\end{array}\right] .
$$

Now it is clear that, at the normal incidence $\left(k_{x 0}=0\right)$, the $R$ matrix reduces to the rotation matrix $R_{0}(\varphi)$, in accordance with the convention of the standard representation (see Eq. (9)). At $k_{x 0}=0$, the common factor $n_{o}^{2} \cos \theta$ in $R$ can be omitted because it cancels when being substituted into Eq. (13).

Substituting Eq. (15) into Eq. (13), one obtains the explicit form of the EJM:

$$
J=e^{-i k \bar{k} z}\left[\begin{array}{cc}
\cos \frac{\Delta k z}{2}-i \frac{1-\bar{s}^{2}+\Delta s^{2}}{1+\bar{s}^{2}-\Delta s^{2}} \sin \frac{\Delta k z}{2} & \frac{2 i \bar{s}+i \Delta s}{1+\bar{s}^{2}-\Delta s^{2}} \sin \frac{\Delta k z}{2} \\
\frac{2 i \bar{s}-i \Delta s}{1+\bar{s}^{2}-\Delta s^{2}} \sin \frac{\Delta k z}{2} & \cos \frac{\Delta k z}{2}+i \frac{1-\bar{s}^{2}+\Delta s^{2}}{1+\bar{s}^{2}-\Delta s^{2}} \sin \frac{\Delta k z}{2}
\end{array}\right],
$$

where

$$
\begin{aligned}
& \bar{k}=\frac{\left(k_{2}+k_{1}\right)}{2}, \Delta k=k_{2}-k_{1}, \bar{s}=\frac{\left(s_{1}-s_{2}\right)}{2}, \Delta s=s_{2}+s_{1}, \\
& s_{1}=\bar{s}+\frac{\Delta s}{2}=\frac{S_{11}}{S_{21}}=\frac{k_{1}^{2}}{n_{0}^{2}} \cot \varphi-\frac{k_{2} k_{x 0} \tan \theta}{\sin \varphi n_{0}^{2}}, \text { and } s_{2}=-\bar{s}+\frac{\Delta s}{2}=\frac{-S_{22}}{S_{12}}=\cot \varphi-\frac{k_{x 0} \tan \theta}{k_{1} \sin \varphi} .
\end{aligned}
$$

\section{EJM in the principal coordinate system}

It is seen from Eq. (16) that the form of the matrix $J$ is similar to that of the GJM $M$ (see also Eq. (23) below). However, one has to recall that the EJM appearing in Eq. (16) is written in the $k$-coordinate system, while $M$ is expressed in the principal $n$-coordinate system. To compare the $J$ and $M$ matrices, one has to transform the former to the $n$-coordinate system. Mathematically this transformation may be written as

$$
J^{0}=R_{0}^{-1}(\varphi) J R_{0}(\varphi)
$$

where $J^{0}$ is the matrix $J$ expressed in the principal coordinate system and $R_{0}$ the rotation matrix given by Eq. (9). After this rotation in the $n$-coordinate system, $-\varphi$ becomes the azimuthal angle of the incident ray (see Fig. 1). Accounting for Eqs. (9) and (13), one can symbolically rewrite $J^{0}$ (see Eq. (17)) as $J^{0}=U G_{0} U^{-1}$, where

$$
U=R_{0}^{-1}(\varphi) R=\left[\begin{array}{cc}
k_{1}^{2} \cos \theta-k_{2} k_{x 0} \sin \theta \cos \varphi+k_{x 0}^{2} \cos \theta \sin ^{2} \varphi & -k_{1} k_{x 0} \sin \theta \sin \varphi \\
k_{2} k_{x 0} \sin \theta \sin \varphi+k_{x 0}^{2} \cos \theta \sin \varphi \cos \varphi & k_{1}^{2} \cos \theta-k_{1} k_{x 0} \sin \theta \cos \varphi
\end{array}\right] .
$$

In the explicit form we have

$$
J^{0}=e^{-i \bar{k} z}\left[\begin{array}{cc}
U_{11} U_{11}^{-1} e^{-i \frac{\Delta k}{2} z}+U_{12} U_{21}^{-1} e^{i \frac{\Delta k}{2} z} & U_{11} U_{12}^{-1} e^{-i \frac{\Delta k}{2} z}+U_{12} U_{22}^{-1} e^{i \frac{\Delta k}{2} z} \\
U_{21} U_{11}^{-1} e^{-i \frac{\Delta k}{2} z}+U_{22} U_{21}^{-1} e^{i \frac{\Delta k}{2} z} & U_{21} U_{12}^{-1} e^{-i \frac{\Delta k}{2} z}+U_{22} U_{22}^{-1} e^{i \frac{\Delta k}{2} z}
\end{array}\right] .
$$

With the matrix $U$ given by Eq. (18), we find the following relations: 


$$
\left\{\begin{array}{l}
U_{11} U_{11}^{-1}=U_{22} U_{22}^{-1}=1-\tau_{11} \\
U_{12} U_{21}^{-1}=U_{21} U_{12}^{-1}=\tau_{11} \\
U_{12} U_{22}^{-1}=-U_{11} U_{12}^{-1}=\tau_{12} \\
U_{22} U_{21}^{-1}=-U_{21} U_{12}^{-1}=\tau_{21}
\end{array},\right.
$$

where

$$
\left\{\begin{array}{l}
\tau_{11}=\frac{k_{1} k_{x 0}^{2} \sin \varphi}{D}\left(k_{2} \sin ^{2} \theta \sin \varphi+k_{x 0} \sin \theta \cos \theta \cos \varphi\right) \\
\tau_{12}=\frac{k_{1} k_{x 0} \sin \varphi}{D}\left(k_{2} k_{x 0} \sin ^{2} \theta \cos \varphi-k_{1}^{2} \cos \theta \sin \theta-k_{x 0}^{2} \cos \theta \sin \theta \sin ^{2} \varphi\right) \\
\tau_{21}=\frac{k_{1} k_{x 0} \sin \varphi}{D}\left(k_{x 0} \sin \theta \cos \varphi-k_{1} \cos \theta\right)\left(k_{x 0} \cos \theta \cos \varphi+k_{2} \sin \theta\right) \\
D=\left(k_{1}^{2} \cos \theta \cos \varphi-k_{1} k_{x 0} \sin \theta\right)\left(k_{1}^{2} \cos \theta \cos \varphi-k_{2} k_{x 0} \sin \theta\right)+k_{1}^{2} n_{0}^{2} \cos ^{2} \theta \sin ^{2} \varphi
\end{array}\right.
$$

Substituting Eqs. (20) into Eqs. (19), we rewrite $J^{0}$ resulting in

$$
J^{0}=e^{-i \overline{k z}}\left[\begin{array}{cc}
\cos \frac{\Delta k z}{2}-i\left(1-2 \tau_{11}\right) \sin \frac{\Delta k z}{2} & (2 i \tau+i \Delta \tau) \sin \frac{\Delta k z}{2} \\
(2 i \tau-i \Delta \tau) \sin \frac{\Delta k z}{2} & \cos \frac{\Delta k z}{2}+i\left(1-2 \tau_{11}\right) \sin \frac{\Delta k z}{2}
\end{array}\right],
$$

where $\tau=\left(\tau_{12}+\tau_{21}\right) / 2, \Delta \tau=\tau_{12}-\tau_{21}$, i.e. $\tau_{12}=\tau+\Delta \tau / 2, \tau_{21}=\tau-\Delta \tau / 2$.

\section{EJM versus GJM}

Let us write out the $M$ matrix in the following form:

$$
M=e^{-i \chi z}\left[\begin{array}{cc}
\cos \frac{T}{2} z-i \frac{L B-i L D}{T} \sin \frac{T}{2} z & \frac{C B-i C D-i\left(L B_{J}-i L D_{J}\right)}{T} \sin \frac{T}{2} z \\
\frac{-C B+i C D-i\left(L B_{J}-i L D_{J}\right)}{T} \sin \frac{T}{2} z & \cos \frac{T z}{2}+i \frac{L B-i L D}{T} \sin \frac{T}{2} z
\end{array}\right] .
$$

Now that the both matrices are expressed in the same $n$-coordinate system, the EJM $J^{0}$ (see Eqs. (22)) can be compared to the GJM M. To be explicit, we have used the notations in Eq. (23) similar to those introduced in Ref. [21]. Both the notation and the analogies between the EJM designed for the oblique incidence and the GJM used for the normal incidence are summarised in Table 1. Equating the corresponding real and imaginary parts of Eqs. (22) and (23) results in $\bar{\kappa}=0, L D=0, L D_{J}=0, C B=0, T=\Delta k, L B / T=1-2 \tau_{11}, L B_{J} / T=-2 \tau, C D / T=-\Delta \tau$.

As expected, the mean absorption, the Jones dichroism and the circular birefringence are absent since we consider non-absorbing, non-gyrotropic crystal plates. The phase retardation due to the linear birefringence is represented by the term $L B=\left(1-2 \tau_{11}\right) \Delta k$. At $k_{x 0}=0$, i.e. for the normal light incidence, we have $\tau_{11}=0$ and the linear phase retardation reduces to its conventional form, $L B=2 \pi \Delta n / \lambda$. Due to the inequality $\tau_{12} \neq \tau_{21} \neq 0$, the off-diagonal components $J_{12}^{0}=2 i \tau_{12} \sin (\Delta k z / 2)$ and $J_{21}^{0}=2 i \tau_{21} \sin (\Delta k z / 2)$ can be expressed respectively as $J_{12}^{0}=2 i(\tau+\Delta \tau / 2) \sin (\Delta k z / 2)$ and $J_{21}^{0}=2 i(\tau-\Delta \tau / 2) \sin (\Delta k z / 2)$. This is why the factor $-2 \tau \Delta k$ plays a role of the Jones birefringence $L B_{J}$ (or $g_{45}$ in the Jones' notation [7]) and $-\Delta \tau \Delta k$ of the circular dichroism. 
Table 1. An analogy between the EJM and the GJM

\begin{tabular}{|c|c|c|c|}
\hline \multirow[t]{2}{*}{ Optical parameter } & \multicolumn{3}{|c|}{ Symbols and definitions } \\
\hline & GJM [21] & EJM (Eq. 22) & GJM [7] \\
\hline $\begin{array}{l}\text { Mean complex wave } \\
\text { number }\end{array}$ & $\chi=\frac{2 \pi}{\lambda}(n-i \kappa)$ & reduces to $\bar{k}$ & $-T_{\bar{N}}=k+i \eta$ \\
\hline $\begin{array}{l}\text { Mean phase retardation } \\
\text { per unite length }\end{array}$ & $\frac{2 \pi}{\lambda} n$ & $\bar{k}=\frac{k_{z 1}+k_{z 2}}{2}$ & $\eta=\frac{2 \pi}{\lambda} n$ \\
\hline $\begin{array}{l}\text { Mean absorption } \\
\text { coefficient }\end{array}$ & $\frac{2 \pi}{\lambda} \kappa$ & 0 & $\kappa=\frac{2 \pi}{\lambda} k$ \\
\hline $\begin{array}{l}\text { Total phase retardation } \\
\text { per unite length }\end{array}$ & $T$ & $\Delta k=k_{2}-k_{1}$ & $\Gamma$ \\
\hline Linear birefringence & $L B=\frac{2 \pi}{\lambda}\left(n_{y}-n_{x}\right)$ & $\left(1-2 \tau_{11}\right) \Delta k$ & $g_{0}=\frac{1}{2}\left(\eta_{y}-\eta_{x}\right)$ \\
\hline Linear dichroism & $L D=\frac{2 \pi}{\lambda}\left(\kappa_{y}-\kappa_{x}\right)$ & 0 & $p_{0}=\frac{1}{2}\left(\kappa_{y}-\kappa_{x}\right)$ \\
\hline Circular birefringence & $C B=\frac{2 \pi}{\lambda}\left(n_{r}-n_{l}\right)$ & 0 & $\omega_{0}=\frac{1}{2}\left(\eta_{r}-\eta_{l}\right)$ \\
\hline Circular dichroism & $C D=\frac{2 \pi}{\lambda}\left(\kappa_{r}-\kappa_{l}\right)$ & $-\Delta \tau \Delta k$ & $\delta=\frac{1}{2}\left(\kappa_{r}-\kappa_{l}\right)$ \\
\hline Jones birefringence & $L B_{J}=\frac{2 \pi}{\lambda}\left(n_{45}-n_{135}\right)$ & $-2 \tau \Delta k$ & $g_{45}=\frac{1}{2}\left(\eta_{45}-\eta_{135}\right)$ \\
\hline Jones dichroism & $L D_{J}=\frac{2 \pi}{\lambda}\left(\kappa_{45}-\kappa_{135}\right)$ & 0 & $p_{45}=\frac{1}{2}\left(\kappa_{45}-\kappa_{135}\right)$ \\
\hline
\end{tabular}

The physical meaning of the Jones birefringence $L B_{J}$ has been discussed by Jones [7] using a transparent, non-gyrotropic $\left(\bar{\kappa}=0, L D=0, L D_{J}=0, C B=0, C D=0\right)$ crystal as an illustrative example. For this case the $M$ matrix reduces to

$$
M_{t}=e^{-i \frac{2 \pi}{\lambda} \bar{n} z}\left[\begin{array}{cc}
\cos \frac{T_{t} z}{2}-\frac{i L B}{T_{t}} \sin \frac{T_{t}}{2} z & \frac{-i L B_{J}}{T_{t}} \sin \frac{T_{t}}{2} z \\
\frac{-i L B_{J}}{T_{t}} \sin \frac{T_{t}}{2} z & \cos \frac{T_{t} z}{2}+\frac{i L B}{T_{t}} \sin \frac{T_{t}}{2} z
\end{array}\right],
$$

where $T_{t}=\sqrt{L B^{2}+L B_{J}^{2}}$. The matrix $M_{t}$ can be diagonalised by its rotation through an angle $\alpha$ such that $\tan 2 \alpha=L B_{J} / L B$, with $L B=T_{t} \cos 2 \alpha$ and $L B_{J}=T_{t} \sin 2 \alpha$. In terms of $\alpha$, the matrix $M_{t}$ becomes as

$$
M_{t}=e^{-i \frac{2 \pi}{\lambda} \bar{n} z}\left[\begin{array}{cc}
\cos \frac{T_{t} z}{2}-i \cos 2 \alpha \sin \frac{T_{t}}{2} z & -i \sin 2 \alpha \sin \frac{T_{t}}{2} z \\
-i \sin 2 \alpha \sin \frac{T_{t}}{2} z & \cos \frac{T_{t} z}{2}+i \cos 2 \alpha \sin \frac{T_{t}}{2} z
\end{array}\right]
$$

After the rotation, the matrix $M_{t}$ transforms into

$$
M_{t 0}=R_{0}^{-1}(\alpha) M_{t} R_{0}(\alpha)=e^{-i \frac{2 \pi}{\lambda} \bar{n} z}\left[\begin{array}{cc}
e^{-\frac{T_{t}}{2} z} & 0 \\
0 & e^{\frac{T_{t}}{2} z}
\end{array}\right]
$$


As a consequence, a transparent, non-gyrotropic crystal plate with a nonzero Jones birefringence is equivalent to a crystal plate with the phase retardation $T_{t}=\sqrt{L B^{2}+L B_{J}^{2}}$ and its axes rotated by the angle $2 \alpha=\arctan \left(L B_{J} / L B\right)$ with respect to the $X$ axis. In the other cases comprising absorbing or/and gyrotropic crystals, $M$ can be diagonalised by no rotation of the coordinate system. Naturally, the EJM matrix that appears in Eq. (22) also cannot be diagonalised by rotations of the coordinate system because of nonzero $\Delta \tau$. The both terms $2 \tau \Delta k$ and $\Delta \tau \Delta k$ make the offdiagonal components of the EJM nonzero at $\varphi \neq 0$, owing to symmetry breaking that occurs for the light propagation problem under condition of the oblique incidence. The origin of these two terms is rooted in the form of the matrix $S$. Indeed, $S$ and consequently $R$ are not orthogonal at $\varphi \neq 0, \theta \neq 90^{\circ}$ and, as a result, the matrix $J$ (see Eq. 22) is not symmetric at $\varphi \neq 0, \theta \neq 90^{\circ}$.

Presence of the term $\Delta \tau \Delta k$ reveals a problem hidden in the form of the $S$ matrix. Although the crystal slab is transparent (no light absorption and so no linear or circular dichroisms are assumed), it is easy to show that the term $\Delta \tau \Delta k$ affects the amplitude of the transmitted light even with no analyser placed behind the crystal and produces a circular dichroism. The losses due to the term $\Delta \tau \Delta k$ could be associated with light reflection, which effectively plays a role of light absorption for the transmitted light, decreasing its amplitude. However, the Lien's approach declares that the light reflection is neglected. This problem might be also coupled with the form of the boundary conditions, which have been applied only to the electric field vector by the author of the work [11]. It is clear from Eq. (23) that for a transparent, non-gyrotropic crystal and under neglect of the light reflection, the Jones matrix has to be symmetric. We are therefore led to conclude that the Lien's EJM given by Eqs. (1)-(6) is in fact not free of the reflection effects, in spite of the initial assumptions made in the study [11]. This problem might be a cause for the errors in the light transmittance calculations produced by the Lien's matrix at high $\left(>45^{\circ}\right)$ incidence angles (see Section 1 and Ref. [12]). It would be of both practical and fundamental interests to check whether the zeroing of the term $\Delta \tau \Delta k$ would decrease the errors occurred in calculations of the optical transmittance of liquid crystal displays.

\section{Homeotropic cell}

It is readily understood that the polarisation state of light transmitted trough crystal is not uniform for the case of conical incident beam. It has been recognised by Nye for the first time [22] that a nonuniform polarisation field might bear polarisation singularities. Among these, one can recall so-called $C$-points, where the light wave is circularly polarised so that the azimuth of the light polarisation ellipse is undefined, and $L$-lines, where the light wave is linearly polarised and so the chirality (left- or right-handed) of the ellipse is undefined. A detailed analysis of the field of polarisation ellipses observed in the conoscopic pattern has been performed analytically by Kiselev et al. [23] (see also references therein) for the case of homeotropic cell and then computed numerically for both tilted and planar cells. An explicitly simple form of the EJM presented above (see Eq. (22)) allows one for analytical consideration of the polarisation ellipse field for any orientations of the optic axis.

Recently it has been claimed [17] that from the singularities mentioned above one can extract a true optical vortex with zero intensity and undefined phase in its core, provided that a paraxial light beam propagates through a slab of uniaxial crystal, with the optic axis and the beam axis directed along the slab normal. Below we will show that, for a crystal slab with its optic axis 
directed along the slab normal (a so-called homeotropic cell), the EJM produces the Jones vector in the form of obtained earlier by the other authors in the paraxial approximation (see [17] and references herein).

For a particular case of the homeotropic cell $\left(\theta=90^{\circ}\right)$, the parameters $\tau_{11}, \tau_{12}$ and $\tau_{21}$ reduce to

$$
1-2 \tau_{11}=\cos 2 \varphi, 2 \tau_{12}=2 \tau_{21}=\sin 2 \varphi,
$$

and the EJM transforms to

$$
J^{H}=e^{-i \overline{k z}}\left[\begin{array}{cc}
\cos \frac{\Delta k z}{2}-i \cos 2 \varphi \sin \frac{\Delta k z}{2} & i \sin 2 \varphi \sin \frac{\Delta k z}{2} \\
i \sin 2 \varphi \sin \frac{\Delta k z}{2} & \cos \frac{\Delta k z}{2}+i \cos 2 \varphi \sin \frac{\Delta k z}{2}
\end{array}\right] .
$$

Here $J^{H}$ is identical to $M_{t}$ (see Eq. (25)) with the accuracy to the signs which can be adjusted while replacing $-\varphi$ by $\alpha$. It is clear that $J^{H}$ is diagonalised by the rotation around the angle $-\varphi$. We recall that $-\varphi$ becomes the azimuth of the incident light ray after the rotation of the coordinate system given by Eq. (17).

Let the incident beam be circularly polarised, with the electric field

$$
E_{0}=\frac{1}{\sqrt{2}}\left[\begin{array}{c}
1 \\
\pm i
\end{array}\right] \text {. }
$$

Then with the form of $J^{H}$ given by Eq. (28), the electric field vector of the light passed through the crystal has the following components:

$$
\left\{\begin{array}{l}
E_{x}=\cos \frac{\Delta k z}{2}+i e^{ \pm i 2 \varphi} \sin \frac{\Delta k z}{2} \\
E_{y}= \pm i\left(\cos \frac{\Delta k z}{2}-i e^{ \pm i 2 \varphi} \sin \frac{\Delta k z}{2}\right)
\end{array}\right.
$$

According to Ref. [17], the factor $e^{ \pm i 2 \varphi}$ is at the origin of optical singularities with the topological charge \pm 2 . Here we do not explore this problem in a much detail, but instead send a reader to Refs. [17, 24-27].

\section{Conclusion}

Starting from the form of the EJM suggested by Lien for the oblique light incidence, we have derived its compact representation, which looks rather handy for further analytical calculations. Our representation of the EJM has the form of the GJM, with nonzero imaginary parts appearing in its off-diagonal elements. A close analogy between these two matrices implies that the terms $2 \tau \Delta k$ and $\Delta \tau \Delta k$ of the EJM play parts of the Jones birefringence and circular dichroism, respectively. The both terms are nonzero in case of $\varphi \neq 0$. Were $\Delta \tau$ equal to zero the EJM could be diagonalised as expected for a non-gyrotropic, non-absorbing crystal under the condition of the light reflection neglected. The presence of the term $\Delta \tau$ that could be treat as a circular dichroism reveals a problem hidden in the Lien's extended rotation matrix $S$. This problem has been uploaded in the $S$ matrix by neglecting the light reflection and putting incomplete boundary conditions in the work [11] that deal only with the electric field vector. For the incidence angles smaller than $45^{\circ}$, the term $\Delta \tau$ approaches zero and the EJM becomes accurate enough for describing light propagation through crystalline slabs at the oblique incidence. 
A simple analytical form of the EJM derived in this work allows for analytical studies of the conoscopic patterns arising behind crystalline slabs, including polarisation-resolved conoscopic mapping of the singular $C$-points and $L$-lines, which has recently been performed only numerically. For the homeotropic liquid-crystal cell, our representation of the EJM reduces to the form that gives the Jones vector identical to that obtained by the other authors in the paraxial approximation. The latter is at the origin of optical singularities generated using optically uniaxial crystals.

\section{Acknowledgement}

The authors are grateful to Dr. Krupych O. M. for many fruitful discussions. The work was supported through the grant 0109U001062 from Ministry of Education and Science of Ukraine.

\section{References}

1. Jones R, 1941. A new calculus for the treatment of optical systems. I. Description and discussion of the calculus. J. Opt. Soc. Amer. 31: 488-493.

2. Jones R, 1941. A new calculus for the treatment of optical systems. II. Proof of three general equivalence theorems. J. Opt. Soc. Amer. 31: 493-499.

3. Jones R, 1941. A new calculus for the treatment of optical systems. III. The Sohncke theory of optical activity. J. Opt. Soc. Amer. 31: 500-503.

4. Jones R, 1942. A new calculus for the treatment of optical systems. IV. J. Opt. Soc. Amer. 32: 486-493.

5. Jones R, 1947. A new calculus for the treatment of optical systems. V. A more general formulation, and description of another calculus. J. Opt. Soc. Amer. 37: 107-110.

6. Jones R, 1947. A new calculus for the treatment of optical systems. VI. Experimental determination of the matrix. J. Opt. Soc. Amer. 37: 110-112.

7. Jones R, 1948. A new calculus for the treatment of optical systems. VII. Properties of the Nmatrices. J. Opt. Soc. Amer. 38: 671-685.

8. Yeh P, 1982. Extended Jones matrix method. J. Opt. Soc. Amer. 72: 507-513.

9. Gu C and Yeh P, 1993. Extended Jones matrix method. II. J. Opt. Soc. Amer. A. 10: 966-973.

10. Lien A, 1990. Extended Jones matrix representation for the twisted nematic liquid crystal display at oblique incidence. App. Phys. Lett. 57: 2767-2769.

11. Lien A, 1997. A detailed derivation of extended Jones matrix representation for twisted nematic liquid crystal displays. Liq. Cryst. 22: 171-175.

12. Yu F H and Kwok H S, 1999. Comparison of extended Jones matrices for twisted nematic liquid crystal displays at oblique angles of incidence. J. Opt. Soc. Amer. A. 16: 2772-2780.

13. Wöhler H, Hass G, Fritsch M and Mlynski D A, 1988. Faster 4x4 matrix method for inhomogeneous uniaxial media. J. Opt. Soc. Amer. A. 5: 1554-1557.

14. Berreman D, 1972. Optics in stratified and anisotropic media: 4x4 matrix formulation. J. Opt. Soc. Amer. 62: 502-510.

15. Berreman D, 1973. Optics in smoothly varying anisotropic planar structure: application to liquid crystal twist cells. J. Opt. Soc. Amer. 63: 1374-1380.

16. Lien A and Chen C J, 1996. A new $2 \times 2$ matrix representation for twisted nematic liquid crystal displays at oblique incidence. Jpn. J. Appl. Phys. 35: L1200-L1203.

17. Volyar A and Fadeyeva T, 2003. Generation of singular beams in uniaxial crystals. Opt. Spectrosc. 94: 235-244. 
18. Nye J F and Berry M V, 1974. Dislocations in wave trains. Proc. Roy. Soc. Lond. A 336: $165-190$.

19. Soskin M S and Vasnetsov M V, 2001. Singular beams. Progr. Opt. 42: 219-276.

20. Azzam R M A and Bashara N M, Ellipsometry and polarized light. Oxford: North-Holland Publishing Company (1977).

21. Artega $\mathrm{O}$ and Canillas A, 2010. Analytic inversion of the Mueller-Jones polarization matrices for homogeneous media. Opt. Lett. 35: 559-561.

22. Nye J F, Natural focusing and fine structure of light. Bristol and Philadelphia: IOP Publishing (1999).

23. KiselevA D, Vovk R G, Egorov R I and Chigrinov V G, 2008. Polarization-resolved angular patterns of nematic liquid crystal cells: Topological events driven by incident light polarization. Phys. Rev. A. 78: 033815.

24. Volyar A V, Shvedov V G and Fadeeva T A, 2002. Optical vortex generation and Jones vector formalism. Opt. Spectrosc. 93: 267-272.

25. Volyar A V, Fadeyeva T A and Egorov Yu A, 2002. Vectorial singularities of Gaussian beams in uniaxial crystals: generation of optical vortices. Pis'ma v Zhurn. Tekhn. Fiz. 28: 70-77.

26. Volyar A V and Fadeyeva T A, 2003. Optical vortices in crystals: birth, annihilation and splitting of polarization umbilics. Pis'ma v Zhurn. Tekhn. Fiz. 29: 58-64.

27. Volyar A, Shvedov V, Fadeyeva T, Desyatnikov A S, Neshev D N, Krolikowski W and Kivshar Y S, 2006. Generation of single-charge optical vortices with an uniaxial crystal. Opt. Express. 14: 3724-3729.

Nastishin Yu. A. and Nastyshyn S. Yu., 2011. Explicit representation of extended Jones matrix for oblique light propagation through a crystalline slab. Ukr.J.Phys.Opt. 12: 191-201.

Анотація. Отримано компактне представлення узагальненої матриці Джонса (УМД), запропонованої Ліеном [Lien A, 1997. Liq. Cryst., 22, 2: 171 - 175] для похилого падіння світла. Наме представлення УМД є зручним для аналітичних обчислень та має форму загальної матриџі Джонса з ненульовою уявною частиною недіагональних елементів. Обговорено аналогію між иими двома матрииями. Для кристалічної пластинки з оптичною віссю вздовж нормалі до пластинки наше представлення УМД зводиться до форми, яка приводить до вектора Джонса, отриманого іншими авторами в параксіальному наближенні. 